\title{
SOME PROPERTIES OF THE FEJER POLYNOMIALS
}

\author{
FRITZ HERZOG AND GEORGE PIRANIAN ${ }^{1}$
}

1. Introduction. The polynomials

$$
P_{n}(z)=\frac{1}{n}+\frac{z}{n-1}+\cdots+\frac{z^{n-1}}{1}-\frac{z^{n}}{1}-\frac{z^{n+1}}{2}-\cdots-\frac{z^{2 n-1}}{n}
$$

were introduced, by way of their real and imaginary parts on the unit circle $C$, by Fejér $[6 ; 7]$. Fejér showed that the number $2+\pi$ is an upper bound for the modulus both of the real and of the imaginary part of $P_{n}$ on $C(n=1,2, \cdots)$; a very simple proof that the polynomials $P_{n}$ are uniformly bounded on $C$ is given in $[3$, p. 43]. In view of recent applications of the Fejér polynomials in the study of Taylor series (see, for example, $[3 ; 4 ; 8]$ ), we have undertaken an investigation of their least upper bound on $C$ (see $\$ 3$ ) and of the distribution of their zeros (see \$2).

Elementary considerations show that $\lim _{n \rightarrow \infty} n P_{n}(z)=1 /(1-z)$ for $|z|<1$, and that the convergence is uniform in every disc $|z| \leqq r<1$. From this and the fact that the reciprocal of every zero of $P_{n}$ is also a zero of $P_{n}$, it follows that the zeros of $P_{n}$ lie on or near the circle $C$. The theorem of Jentzsch and Szegö $[9 ; 11]$ implies further that the arguments of the zeros are uniformly distributed in the interval $[0,2 \pi]$. Somewhat stronger results on the distribution of the arguments could be obtained by applying a theorem of Erdös and Turán [5, p. 106]; but by using a method which involves nothing deeper than Rouchés theorem, we prove that each of the $n-1$ sectors

$$
z=r e^{i \theta}, \quad \frac{(2 k-1) \pi}{n}<\theta<\frac{(2 k+1) \pi}{n} \quad(k=1,2, \cdots, n-1)
$$

contains precisely two zeros of $P_{n}$.

2. The zeros of the Fejér polynomials. We shall state and prove various lemmas concerning the zeros of the polynomials $P_{n}$. At the end of the section, the contents of the lemmas will be gathered into a theorem.

Received by the editors June 2, 1955.

${ }^{1}$ G. Piranian's contributions to this 'paper were made under Contract DA 20-018ORD-13585 between the Office of Ordnance Research and the Engineering Research Institute of the University of Michigan. 
Lemma 1. The point $z=1$ is the only positive zero of $P_{n}$. The reciprocal and the complex conjugate of every zero of $P_{n}$ are also zeros of $P_{n}$.

This lemma follows at once from Descartes' rule of signs, from the fact that the coefficients of the polynomial $P_{n}$ are real, and from the relation $P_{n}\left(z^{-1}\right)=-z^{-2 n+1} P_{n}(z)$.

Lemma 2. Except for $z=1, P_{n}$ has no zeros on the unit circle.

It is easily verified that

$$
P_{n}\left(e^{i \theta}\right)=-2 i e^{i(n-1 / 2) \theta} C(n, \theta),
$$

where

$$
C(n, \theta)=\sum_{k=1}^{n} \frac{\sin (k-1 / 2) \theta}{k} .
$$

Using the identity in $[10, \S \mathrm{VI}$, Problem 17, p. 78$]$ and Abel's summation, we obtain the formula

$$
C(n, \theta)=\frac{1}{\sin (\theta / 2)}\left\{\frac{\sin ^{2}(n \theta / 2)}{n}+\sum_{k=1}^{n-1} \frac{\sin ^{2}(k \theta / 2)}{k(k+1)}\right\} .
$$

The lemma follows from the fact that the right member is positive for $0<\theta<2 \pi$.

In the sequel, it will be useful to deal with the function

$$
W_{n}(z)=z^{-n}(1-z) P_{n}(z)=\frac{z^{n}+z^{-n}}{n}-2+\sum_{k=1}^{n-1} \frac{z^{k}+z^{-k}}{k(k+1)} .
$$

LEMMA 3. Each of the $n$ sectors

$$
(2 k-1) \pi / n<\arg z<(2 k+1) \pi / n \quad(k=0,1, \cdots, n-1)
$$

contains exactly two zeros of $W_{n}(z)$.

Let

$$
f_{n}(z)=\frac{z^{n}+z^{-n}}{n}-2, \quad g_{n}(z)=\sum_{k=1}^{n-1} \frac{z^{k}+z^{-k}}{k(k+1)} .
$$

If $z$ lies on one of the rays

$$
\arg z=\frac{(2 k+1) \pi}{n} \quad(k=0,1, \cdots, n-1),
$$

then, with $|z|=r,\left|f_{n}(z)\right|=\left(r^{n}+r^{-n}\right) / n+2$, and therefore $\left|f_{n}(z)\right|$ $-\left|g_{n}(z)\right| \geqq \psi(r)$, where 


$$
\psi(r)=\frac{r^{n}+r^{-n}}{n}+2-\sum_{k=1}^{n-1} \frac{r^{k}+r^{-k}}{k(k+1)} .
$$

To show that $\psi(r)>0$ for $r>0$, it is sufficient to consider the values $r \geqq 1$. Now $\psi(1)=4 / n>0$; and $\psi^{\prime}(r) \geqq 0$ when $r \geqq 1$, since the function

$$
r \psi^{\prime}(r)=r^{n}-r^{-n}-\sum_{k=1}^{n-1} \frac{r^{k}-r^{-k}}{k+1}
$$

vanishes at $r=1$ while its derivative

$$
r^{-1}\left\{n\left(r^{n}+r^{-n}\right)-\sum_{k=1}^{n-1} \frac{k\left(r^{k}+r^{-k}\right)}{k+1}\right\}
$$

is obviously positive. It follows that

$$
\left|f_{n}(z)\right|>\left|g_{n}(z)\right|
$$

on each of the rays (3). The inequality (4) is also satisfied on the circle $|z|=r_{n}$, provided $r_{n}$ is sufficiently small or sufficiently large. By applying Rouche's theorem to the functions $f_{n}$ and $f_{n}+g_{n}=W_{n}$, with reference to a region which is bounded by two neighboring rays (3) and by arcs of two circles $|z|=r_{n}$ and $|z|=r_{n}^{-1}$, we conclude that $f_{n}$ and $W_{n}$ have the same number of zeros in this region. The lemma now follows from the fact that the zeros of $f_{n}$ are the $2 n$ numbers

$$
\left[n \pm\left(n^{2}-1\right)^{1 / 2}\right]^{1 / n} e^{2 k \pi i / n} \quad(k=0,1, \cdots, n-1) .
$$

LemMa 4. $P_{n}$ has two or no negative zeros, depending on whether $n$ is even or odd.

The function $W_{n}$ has $2 n$ zeros of which two lie at $z=1$. If $n$ is odd, Lemma 3 disposes of the remaining $2 n-2$ zeros. If $n$ is even, Lemma 3 implies that at most two zeros are negative, and Lemmas 1 and 2 imply that at least two zeros are negative.

Lemma 5. If $\epsilon>0$ and $n$ is sufficiently large, the zeros of $P_{n}$ lie in the annulus $\exp \left[-(4+\epsilon) n^{-1} \log n\right]<|z|<\exp \left[(4+\epsilon) n^{-1} \log n\right]$.

It suffices to show that $W_{n}(z) \neq 0$ for $|z| \leqq \exp \left[-(4+\epsilon) n^{-1} \log n\right]$ and $n \geqq n_{0}(\epsilon)$. By $(2), z^{n} W_{n}(z)=1 / n+V_{n}(z)$, where

$$
\begin{aligned}
V_{n}^{\prime}(z)= & \frac{z}{n(n-1)}+\frac{z^{2}}{(n-1)(n-2)}+\cdots+\frac{z^{n-1}}{2 \cdot 1}-2 z^{n} \\
& +\frac{z^{n+1}}{1 \cdot 2}+\frac{z^{n+2}}{2 \cdot 3}+\cdots+\frac{z^{2 n-1}}{(n-1) n}+\frac{z^{2 n}}{n} .
\end{aligned}
$$


We shall now show that $\left|V_{n}(z)\right|<1 / n$ for the values of $z$ indicated above.

Let $\eta=2+\epsilon / 2$. The sum of the moduli of the first $[n / \eta]$ terms on the right-hand side of (5) is less than

$$
\frac{1}{n-[n / \eta]}-\frac{1}{n} \leqq \frac{1}{n-n / \eta}-\frac{1}{n}=\frac{1}{n(\eta-1)}
$$

Since the remaining terms have coefficients of modulus at most 2 , the modulus of their sum is at most

$$
\frac{2|z|^{n / \eta}}{1-|z|} \leqq \frac{2 n^{-2}}{1-\exp \left[-2 \eta n^{-1} \log n\right]}=\frac{1+o(1)}{\eta n \log n} .
$$

It follows that, for large $n$,

$$
\left|V_{n}(z)\right| \leqq \frac{1}{n(\eta-1)}+O\left(\frac{1}{n \log n}\right)<\frac{1}{n},
$$

and the lemma is proved.

Lемма 6. If $n$ is even, the two negative zeros of $P_{n}$ are given asymptotically by the formula

$$
z=-[n \log 16+o(n)]^{ \pm 1 / n}
$$

To prove this lemma, we suppose that $W_{n}\left(-r_{n}\right)=0$, with $r_{n}>1$, and we write $r_{n}=n^{p_{n} / n}=\exp \left(p_{n} n^{-1} \log n\right)$. (For the sake of typographical simplicity, we drop the subscript $n$ in the remainder of the proof.)

By Lemma $5, p<5$ when $n$ is sufficiently large. From equation (2) we obtain the relation

$$
n^{p-1}+n^{-p-1}=h(r)
$$

where

(7) $h(r)=2+\frac{r+r^{-1}}{1 \cdot 2}-\frac{r^{2}+r^{2}}{2 \cdot 3}+\frac{r^{3}+r^{-3}}{3 \cdot 4}-\cdots+\frac{r^{n-1}+r^{1-n}}{(n-1) n}$.

The proof of the lemma hinges on an effective computation of $h(r)$. For the purpose of this computation, we divide the right-hand member of (7) into two sections. The first section contains the number 2 and the first $[3 n /(p \log n)]$ terms that follow. The $k$ th of these terms has the modulus $2 / k(k+1)+\epsilon_{k}$, where

$$
\epsilon_{k}=\frac{2 \cosh \left(k p n^{-1} \log n\right)-2}{k(k+1)} .
$$


Since $\cosh u<1+2 u^{2}$ when $0<u \leqq 3$,

$$
0<\epsilon_{k}<\frac{4\left(k p n^{-1} \log n\right)^{2}}{k(k+1)}<4\left(p n^{-1} \log n\right)^{2},
$$

and

$$
\sum_{k \leqq 3 n /(p \log n)} \epsilon_{k}<\frac{3 n}{p \log n} \cdot 4\left(p n^{-1} \log n\right)^{2}=12 p n^{-1} \log n=o(1) .
$$

It follows that the sum of the terms in the first section on the right of (7) is $\log 16+o(1)$.

To show that the sum of the terms in the second section is small, we shall first prove that each term is numerically larger than its predecessor. It will then follow that the sum of the terms in the second section is positive and smaller than the last term. We write

$$
\begin{aligned}
& \frac{r^{k+1}+r^{k-1}}{r^{k+r^{k}}} \cdot \frac{k(k+1)}{(k+1)(k+2)} \\
& \quad=\frac{\cosh \left[(k+1) p n^{-1} \log n\right]}{\cosh \left(k p n^{-1} \log n\right)}\left[1-\frac{2}{k}+\frac{4}{k^{2}}-\cdots\right] .
\end{aligned}
$$

The second factor on the right is greater than $1-2 / k$, that is, greater than $1-(2 / 3) p n^{-1} \log n$. To obtain a lower bound on the first factor, we use the fact that the derivative of $\cosh x$ is an increasing function, and we conclude that the factor is greater than

$$
\begin{aligned}
& \frac{\cosh \left(k p n^{-1} \log n\right)+p n^{-1} \log n \sinh \left(k p n^{-1} \log n\right)}{\cosh \left(k p n^{-1} \log n\right)} \\
& =1+\left(p n^{-1} \log n\right) \tanh \left(k p n^{-1} \log n\right)>1+(\tanh 3) p n^{-1} \log n .
\end{aligned}
$$

Since $\tanh 3>2 / 3$, the ratio between the numerical value of the $(k+1)$ st term and that of the $k$ th term on the right of $(7)$ is greater than 1 when $k>3 n /(p \log n)$, if $n$ is sufficiently large. Since the last term in (7) is less than $\left(n^{p}+1\right) /(n-1) n<3 n^{p-2}$, it follows that

$$
\log 16+o(1) \leqq h(r) \leqq \log 16+3 n^{p-2}+o(1) .
$$

We conclude from equation (6) and the first inequality in (8) that $p>1$; and from (6) and the second inequality in (8) that $p \rightarrow 1$ as $n \rightarrow \infty$. It follows, again from (8), that $h(r)=\log 16+o(1)$. From (6) we now deduce that $r^{n}=n^{p}=n\left[h(r)-n^{-p-1}\right]$, that is,

$$
r=\{n[\log 16+o(1)]\}^{1 / n},
$$

as was to be proved. 
LEMMA 7. If $\epsilon>0$ and $n$ is sufficiently large, the annulus

$$
1-\frac{(2 e)^{1 / 2}-\epsilon}{n} \leqq|z| \leqq 1+\frac{(2 e)^{1 / 2}-\epsilon}{n}
$$

contains no zeros of $P_{n}(z) /(1-z)$.

We write $P_{n}(z) /(1-z)=a_{0}+a_{1} z+a_{2} z^{2}+\cdots+a_{2 n-2} z^{2 n-2}$, where

$$
a_{n-k}=a_{n+k-2}=\frac{1}{n}+\frac{1}{n-1}+\cdots+\frac{1}{k} \quad(k=1,2, \cdots, n) .
$$

We now apply a theorem of Egerváry [2, p. 81] which, slightly specialized for our purpose, reads as follows:

If $a_{m}>0 \quad(0 \leqq m \leqq 2 n-2)$ and if, for some $\rho>1$ and for $m=0$, $1, \cdots, n-2, n, n+1, \cdots, 2 n-2$, the condition

$$
a_{m-1}-\left(\rho+\rho^{-1}\right) a_{m}+a_{m+1}>0
$$

(with the notation $a_{-1}=a_{2 n-1}=0$ ) is satisfied, then the polynomial $\sum_{m} a_{m} z^{m}$ has no zeros in the annulus $\rho^{-1} \leqq|z| \leqq \rho$.

To establish a value of $\rho$ for which our polynomial satisfies the condition (9), we write $\rho=1+b$. Applied to the case $m=n-k$, condition (9) becomes, after some elementary computations,

$$
\frac{b^{2}}{1+b}<\frac{1}{k(k-1)[1 / n+1 /(n-1)+\cdots+1 / k]} \begin{array}{r}
(k=2,3, \cdots, n) .
\end{array}
$$

In order to find an upper bound for the denominator on the right side of (10), we note that this denominator has the value

$$
k(k-1)\left[\frac{1}{n}+\frac{1}{n-1}+\cdots+\frac{1}{k+1}\right]+k-1<k^{2} \log (n / k)+n \text {. }
$$

The maximum of this, for $k \geqq 1$, is $n+n^{2} / 2 e$. It follows that condition (10) is satisfied provided $b^{2}<1 /\left(n+n^{2} / 2 e\right)$; that is, condition (9) is satisfied, for large $n$, if $b=\left((2 e)^{1 / 2}-\epsilon\right) / n$.

The following proposition summarizes the results of this section:

Theorem 1. The polynomial $P_{n}$ has a zero at $z=1$, and it has no other positive zero and no other zero on the unit circle. It has no negative zeros if $n$ is odd; if $n$ is even, it has two negative zeros which are given asymptotically by the formula

$$
z=-1 \pm\left(\frac{\log n}{n}+\frac{\log \log 16}{n}\right)+o\left(\frac{1}{n}\right)
$$


Each of the $n-1$ sectors $(2 k-1) \pi / n<\arg z<(2 k+1) \pi / n(k=1,2$, $\cdots, n-1)$ contains exactly two zeros of $P_{n}$. For $\epsilon>0$ and $n$ sufficiently large, each of the zeros of $P_{n}(z) /(1-z)$ lies in one of the annuli

$$
\begin{aligned}
& 1-\frac{(4+\epsilon) \log n}{n}<|z|<1-\frac{(2 e)^{1 / 2}-\epsilon}{n}, \\
& 1+\frac{(4+\epsilon) \log n}{n}>|z|>1+\frac{(2 e)^{1 / 2}-\epsilon}{n} .
\end{aligned}
$$

3. The maximum modulus of the Fejêr polynomials. We denote by $M_{n}$ the maximum modulus of $P_{n}$ on the unit circle $C$.

Theorem 2. As $n \rightarrow \infty$,

$$
M_{n} \rightarrow 2 \int_{0}^{\pi} \frac{\sin t}{t} d t=3.704 \cdots
$$

From (1) we note that

$$
M_{n}=2 \max _{0 \leqq \theta \leqq \pi} \sum_{k=1}^{n} \frac{\sin (k-1 / 2) \theta}{k} .
$$

The sum on the right is a partial sum of the Fourier series of the function

$$
g(\theta)=\sum_{k=1}^{\infty} \frac{\sin (k-1 / 2) \theta}{k}
$$

which satisfies the relations $g(\theta+4 \pi)=g(\theta), g(-\theta)=-g(\theta)$ and $g(2 \pi-\theta)=g(\theta)$. We write

$$
g(\theta)=\sum_{k=1}^{\infty} \frac{2 \sin (k-1 / 2) \theta}{2 k-1}-\sum_{k=1}^{\infty} \frac{\sin (k-1 / 2) \theta}{k(2 k-1)},
$$

and we observe that the first sum on the right is the well-known Fourier series of the function which takes the values $\pi / 2$ and $-\pi / 2$ in alternate intervals of length $2 \pi$, while the second series on the right converges uniformly for all real $\theta$. Our result now follows from the theory of the Gibbs phenomenon. For a concise statement, we refer the reader to Zygmund $[12, \$ \$ 8.5,8.51]$; interesting graphs and an excellent historical account will be found in [1, Chapter IX].

By a more detailed investigation, we have been able to show that the constant of Theorem 2 is actually an upper bound, and hence the least upper bound, for the maxima $M_{n}(n=1,2, \cdots)$. This in- 
vestigation is based on the representation $C(n, \theta)=A(n, \theta) \cos (\theta / 2)$ $-B(n, \theta) \sin (\theta / 2)$, where

$$
A(n, \theta)=\sum_{k=1}^{n} \frac{\sin k \theta}{k}, \quad B(n, \theta)=\sum_{k=1}^{n} \frac{\cos k \theta}{k} .
$$

The principal properties of these trigonometric polynomials are treated in [10, §VI, Problems 23-28]. The computations involved are so tedious that their publication is not justified.

\section{REFERENCES}

1. H. S. Carslaw, Introduction to the theory of Fourier's series and integrals, 3d ed., London, 1930.

2. E. Egerváry, On a generalisation of a theorem of Kakeya, Acta Univ. Szeged. vol. 5 (1930-1932) pp. 78-82.

3. P. Erdös, F. Herzog and G. Piranian, On Taylor series of functions regular in Gaier regions, Arch. Math. vol. 5 (1954) pp. 39-52.

4. - Sets of divergence of Taylor series and of trigonometric series, Math. Scand. vol. 2 (1954) pp. 262-266.

5. P. Erdös and P. Turán, On the distribution of roots of polynomials, Ann. of Math. (2) vol. 51 (1950) pp. 105-119.

6. L. Fejér, Lebesguesche Konstanten und divergente Fourierreihen, J. Reine Angew. Math. vol. 138 (1910) pp. 22-53.

7. - Sur les singularités de la série de Fourier des fonctions continues, Ann. Ecole Norm. (3) vol. 28 (1911) pp. 63-103.

8. D. Gaier, Über die Summierbarkeit beschränkter und stetiger Potenzreihen an der Konvergenzgrenze, Math. Zeit. vol. 56 (1952) pp. 326-334.

9. R. Jentzsch, Untersuchungen zur Theorie der Folgen analytischer Funktionen, Inaugural-Dissertation, Berlin, 1914.

10. G. Polya and G. Szegö, Aufgaben und Lehrsätze aus der Analysis, vol. II, Berlin, 1925.

11. G. Szegö, Über die Nullstellen von Polynomen, die in einem Kreise gleich mässig konvergieren, Berlin. Math. Ges. Sitzungsber. vol. 21 (1922) pp. 59-64.

12. A. Zygmund, Trigonometric series, Warsaw, 1935.

Michigan State College and UNIVERSITY OF MiCHIGAN 